\title{
ПРОБЛЕМЫ ПОСТРОЕНИЯ МАТЕМАТИЧЕСКИХ МОДЕЛЕЙ
}

Даурбеков С.C.

ГГНТУ им. акад. М.Д. Миллионщикова, г. Грозный, Россия

B статье рассматриваются проблемь построения $и$ анализа математических моделей. Дается понятие сущзности и принципов математического моделирования. Вылелены этапь построения моделей. Показано использование элементарной и высшей математики для решения конкретных практических задач. Выделены компьютерные программные продукты для построения математических моделей.

Ключевые слова: математические модели, этапы и принципы моделирования, компьютерные программные средства.

Использование математических методов в различных сферах человеческой деятельности расширяется из года в год. Невозможно представить качественное исследование проблем без построения математических моделей $[1,5,10]$. Широкое распространение изучения дисциплин математического моделирования по многим специальностям получило и в системе высшего образования.

Сущность моделирования заключается в углублении познания изучаемого процесса, явления; совершенствовании системы, прогнозировании, определении качественных характеристик оригинала и модели и их соответствии.

Укажем на некоторые принципы математического моделирования [11]:

А. Принциип адекватности: модель должна учитывать наиболее существенные стороны исследуемого объекта и отражать его свойства с приемлемой точностью.

В. Принциип простоты и экономичности: модель должна быть достаточно простой для того, чтобы ее использование было эффективно и экономически выгодно. Она не должна быть более сложной, чем это требуется для исследователя.

С. Принциип информацуионной достаточности: при полном отсутствии информации об объекте построить модель невозможно. При наличии полной информации моделирование лишено смысла. Существует уровень информационной достаточности, при достижении которого может быть построена модель системы.

D. Принциип множественности и единства моделей: любая конкретная модель отражает лишь некоторые стороны реальной системы. Для полного исследования необходимо построить ряд моделей, отражающих наиболее существенные стороны исследуемого процесса. Каждая последующая модель должна дополнять и уточнять предыдущую. 
Процесс построения математической модели включает в себя следующие этапы:

1) Всестороннее изучение исследуемого процесса.

2) Идентификация цели модели - определение одного или нескольких показателей, характеризующих суть процесса.

3) Определение и идентификация факторов, влияющих на состоянии исследуемой системы.

4) Сбор и анализ количественных и качественных значений факторов, включенных в исследование.

5) Выбор математической модели и определение математического метода, определяющего входные параметры.

6) Использование компьютерных программ для построения модели.

7) Анализ построенной математической модели, определение адекватности модели.

8) Прогнозные расчеты по модели.

\section{1. Использование элементарных функций}

Математика в виде элементарных арифметических операций применялась в прикладных задачах всегда [8].

На практике многие зависимости могут быть заданы как функции одной переменной: $y=f(x)$.

В качестве примеров функциональных зависимостей можно привести следующие функции, имеющие смысл в соответствующей области значений аргумента.

1.1. Функиия спроса от цүены товара. Обозначим через $x$ цену товара, через у - спрос на товар. Тогда функцию спроса можно выразить аналитически, например, в виде:

$$
y=\frac{100}{x+3} \text { или } y=e^{-x} .
$$

1.2. Суммарная выручка, равная произведению количества проданного товара на цену товара, тоже является функция спроса. Например, если $x-$ спрос, цена $\frac{200}{x+4}$, то выручка $\frac{200 x}{x+4}$.

1.3. Суммарные издержки производства $F$ и средние (удельные) издержки производства (себестоимость) $f$ - функции от объема производства $x$ : $F=F(x), f(x)=F(x) / x$.

1.4. Простые и сложные процентыл. В некоторых задачах, например, в задачах о денежных вкладах в банке, возникает необходимость рассчитать так называемые «простые и сложные проценты».

Если в разные периоды времени $\mathrm{n}_{\mathrm{i}}(\mathrm{i}=1, \ldots, \mathrm{k})$ процент вклада различный и равен $\mathrm{p}_{\mathrm{i}}(\mathrm{i}=1, \ldots, \mathrm{k})$, то формулы простых и сложных процентов запишутся в виде:

$\mathrm{A}_{\mathrm{n}}=\mathrm{A}_{0}\left(1+\mathrm{p}_{1} * \mathrm{n}_{1} / 100+\ldots+\mathrm{p}_{\mathrm{k}} * \mathrm{n}_{\mathrm{k}} / 100\right)-$ простые проценты, 


$$
\begin{aligned}
& \mathrm{A}_{\mathrm{n}}=\mathrm{A}_{0}\left(1+\mathrm{p}_{1} / 100\right)^{\mathrm{n} 1} * \ldots *\left(1+\mathrm{p}_{\mathrm{k}} / 100\right)^{\mathrm{nk}}-\text { сложные проценты, } \\
& \text { где } \mathrm{n}_{1}+\mathrm{n}_{2}+\ldots+\mathrm{n}_{\mathrm{k}}=\mathrm{n} .
\end{aligned}
$$

\section{5. Пределы в социально-экономической сфере}

Пример 1. Между ценой $x$ одного из товаров и спроса $y$ на него установлена. Например, зависимость вида $y=\frac{300}{x+4}$. Как поведет себя функция спроса при неограниченном увеличении цены $(x \rightarrow \infty)$ ?

Решение. $\lim _{x \rightarrow \infty} \frac{300}{x+4}=\frac{300}{\infty}=0$.

Таким образом, при неограниченном росте цен спрос приближается к нулю. (Это простое утверждение доказано математически).

Пример 2. Экономические исследования показывают, что спрос у на товары первой необходимости и спрос $z$ на предметы роскоши зависят от дохода $x$ следующим образом:

$$
\begin{aligned}
y(x)= & \frac{b_{1}\left(x-a_{1}\right)}{x-c_{2}}, x>a_{1} ; \\
z & =\frac{b_{2} x\left(x-a_{2}\right)}{x-c_{2}}, x>a_{2}>a_{1},
\end{aligned}
$$

где $a_{1}, a_{2}-$ уровни доходов, при которых начинаются приобретение тех или иных товаров, $\mathrm{b}_{1}, \mathrm{~b}_{2}, \mathrm{c}_{1}, \mathrm{c}_{2}$ - параметры модели. Функции $y(x)$ и $z(x)$ называются функциями Л. Торнквиста.

Определение пределов функций $y(x)$ и $z(x)$ при $x \rightarrow \infty$ показывает, что при неограниченном увеличении доходов спрос на товары первой необходимости растет до определенного предела, равного $b_{1}$. Поэтому число $b_{1}$ называется уровнем насыщения.

Спрос же на предметы роскоши не имеет уровня насыщения. Он растет даже при неограниченном росте доходов.

\section{6 Анализ статистических данных}

Первоначальный анализ статистических данных заключается в определении таких элементарных показателей как среднее значение, медиана, мода, максимальное и минимальное значения переменных, дисперсия, корреляция и т.п.

\section{2. Дифференциальное и интегральное исчисление}

2.1 Предельные величины

Анализ разнообразных явлений использует ряд предельных величин: предельная скорость изменения технических процессов, предельная стоимость, 
предельные издержки, предельный доход; предельная производительность, предельная полезность, предельная склонность к потреблению. Все эти величины самым тесным образом связаны с понятием производной. В качестве характерного примера рассмотрим предельные издержки.

Пусть $y(x)$ - затраты на изготовление $x$ экземпляров некоторого продукта. Тогда $y^{/}(x)$ выражает скорость изменения затрат при изменении количества продукта.

Пример 1. Определение предельных величин с помощью понятия производной позволяет использовать математический аппарат для доказательства экономических законов. Рассмотрим некоторые применения дифференциального исчисления в экономической теории.

Пусть $x$ - количество реализованного товара, $R(x)$ - функция дохода, $C(x)$ - функция издержек (затрат на производство товара). Вид этих функций зависит от способа производства, оптимизации инфраструктуры и т. п. Обозначим функцию прибыли П $(x)$. Тогда П $(x)=R(x)-C(x)$.

Очевидно, оптимальным уровнем производства является тот, при котором прибыль максимальна, т. е. такое значение выпуска $x$, при котором функция П $(x)$ имеет максимум. Необходимое условие экстремума: $\Pi /(x)=0$ или $R^{/}(x)=C^{/}(x)$, т.е. получаем известное в микроэкономике утверждение: для того, чтобы прибыль была максимальной, необходимо, чтобы предельный доход и предельные издержки были равны.

Пример 2. Определить производительность труда $\mathrm{X}(\mathrm{t})$ некоторой бригады, если известна $\mathrm{Q}(\mathrm{t})$ - функция выпуска продукции этой бригадой в моменты времени $\mathrm{t}$.

Решение. Если применить понятие производной, то решение элементарно: $\mathrm{X}(\mathrm{t})=\mathrm{dQ}(\mathrm{t}) / \mathrm{dt}$

\section{6 Использование интегрального исчисления}

Обратная задача к «Пример 2, пункт 2.1.» - определить выпуск продукции $\mathrm{Q}(\mathrm{t})$ за период времени $\Delta \mathrm{t} €\left[\mathrm{t}_{1}, \mathrm{t}_{2}\right]$, если известна функция $\mathrm{X}(\mathrm{t})$ производительности труда.

Для решения данного примера необходимо использовать интегральное исчисление. Применяя свойства интеграла, получаем: выпуск продукции $\mathrm{Q}(\mathrm{t})$

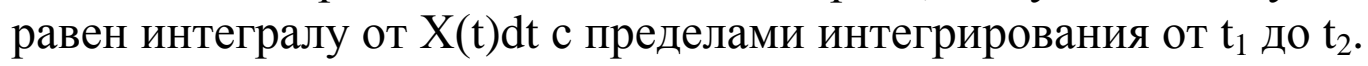

\section{2. Использование задач линейного программирования}

Большой класс разнообразных практических задач при модельном изучении сводятся к моделям линейного программирования (ЛП): оптимизация выпуска продукции, задачи транспортного типа, задача о диете (рационе), задачи комплектности, дробно-линейного программирования, многокритериальные задачи линейного программирования и т. п. [3]. 
В основном, математическая модель ЛП записывается в виде:

$$
\mathrm{Z}(\mathrm{x})=\mathrm{cx} \rightarrow \text { extr, } \quad \mathrm{Ax} \leq \mathrm{b} .
$$

В учебном процессе для построения и анализа математических зависимостей, моделей используются программы Excel, MathCAD [2,4,6,9]. Естественно, имеются и специфические мощные системы Mathlab, SPSS, GRETL и т.д. Но их применение основными пользователями затруднено из-за проблемы лицензирования, высокой стоимости программ.

Элементарные функции, используемые при моделировании можно получить в пакете $\mathrm{f}_{\mathrm{x}}$ - мастер функций (в Excel), в MathCAD - в панели «Calculator».

При определении пределов (предельных значений, асимптот), производных, интегралов лучше использовать функции MathCAD из панелей «Calculus», «Math»: $\lim \mathrm{f}(\mathrm{x}), \operatorname{df}(\mathrm{x}) / \mathrm{dx}$, интг $\left(\mathrm{x}_{1}, \mathrm{x}_{2}, \mathrm{f}(\mathrm{x})\right)$.

Анализ статистических данных $\left(\mathrm{y}_{\mathrm{i}}, \mathrm{x}_{1 \mathrm{i}}, \mathrm{x}_{2 \mathrm{i}}, \ldots, \mathrm{x}_{\mathrm{ki}} ; \mathrm{i}=1, \ldots, \mathrm{n}\right)$ можно осуществить в Excel с помощью функций СРЗНАЧ(х), MЕДИАНА(y), МОДА(х), МАКС(x), МИН(y), ДИСП(x), КОРРЕЛ(x, y), В MathCАD эти функции записываются в виде: mean(x), median(y), moda(x), $\max (\mathrm{x}), \min (\mathrm{y})$, $\operatorname{var}(\mathrm{y}), \operatorname{corr}(\mathrm{x}, \mathrm{y})$.

Основные действия при реализации задачи линейного программирования в Excel осуществляется программой «Поиск решения». «Поиск решения» - эта надстройка Microsoft Office Excel, позволяющая решать оптимизационные задачи.

После ввода исходных данных заполняется таблица программы «Поиск решения»: устанавливаются адреса ячейки целевой функции и вида экстремума (max, min); адреса массива неизвестных, а также адреса системы ограничений. Программа позволяет использовать кроме симплекс-метода и другие численные методы поиска экстремальных точек.

$\mathrm{B}$ MathCAD запись задачи линейного программирования не представляет большого труда. Основные использующиеся команды - $\operatorname{Maximize}(\mathrm{z}, \mathrm{x})$, Minimize(z,x), Given.

Данные программы позволяют без больших осложнений вводить дополнительные ограничения, встречающиеся в реальной деятельности.

\section{3. Нелинейные модели}

На практике для анализа технико-экономических показателей чаще используются нелинейные модели. Надо отметить, что в нелинейном случае не существует единого метода решения поставленных задач. В зависимости от вида задач эти методы подразделяются на: безусловный экстремум, квадратичное программирование, с использованием функции Лагранжа, матрицы Гессе, от скорости сходимости, точности результатов, от получения локальных или глобальных экстремумов и т.п.

Численные методы - основной метод решения нелинейных моделей. Подразделяют три вида методов: нулевого, первого, второго порядков, 
использующих значения целевых функций, первых и вторых частных производных [7].

В программе «Поиск решения» (Excel) возможно получение локального экстремума нелинейных задач с использованием численных методов.

C использованием встроенных функций в MathCAD (частных и вторых частных производных) быстро записывается алгоритм поиска решения нелинейных моделей.

\section{Список литературы}

1. Бояринов А.И., Кафаров В.В. Методы оптимизации в химической технологии. - М.: Химия, 1975.

2. Горчаков А.А., Орлова И.В. Компьютерные экономикоматематические модели. - М.: Издательское объединение «ЮНИТИ», 1995.

3. Даурбеков С. С. Алгоритмы решения линейных задач на экстремум. Грозный, ГГНИ, 2010. - 83 с.

4. Даурбеков С. С., Хадисов М-Р. Б. Математическое моделирование технико-экономических задач в Excel и MathCAD (исправленное и дополненное). Лабораторный практикум - Грозный, ИПЦ ГГНТУ, 2016.

5. Зарубин В.С. Математическое моделирование в технике - М., Изд-во МГТУ им. Баумана, 2001.

6. Коганов В.И. Компьютерные вычисления в средах Excel и MathCAD. - М.: Горячая линия-телеком, 2003. -328 с.

7. Краскевич В. Е., Зеленский К. Х., Гречко В. И. Численные методы в инженерных исследованиях. — Киев: Вища шк., 1986.

8. Математика для экономистов: от арифметики до эконометрики. Под редакцией Н.Ш. Кремера. 2-е издание. - М., ЮРАЙТ, 2010. модели. М., «Компьютер», Из-во: «ЮНИТИ», 1995.

9. Петрунин Ю.Ю. «Решение экономических задач в Ехсеl». Из-во Московского университета, 2001.

10. Солдатенко Л.В. Введение в математическое моделирование строительно-технологических задач. Учебное пособие. Оренбург: ГОУОГУ, 2009. - 161c.

11. Кунин С. Вычислительная физика. М.: Мир, 1992. 518 с. 